\title{
Kearns-Sayre Syndrome
}

National Cancer Institute

\section{Source}

National Cancer Institute. Kearns-Sayre Syndrome. NCI Thesaurus. Code C84798.

A rare mitochondrial myopathy characterized by a progressive limitation of eye movements, leading to immobility and eye drop. It may be associated with muscle weakness, heart block, hearing loss, ataxia and short stature. 